\section{La infección persistente por papilomavirus humanos predice la neoplasia intraepitelial cervical}

La infección por papilomavirus humanos $(\mathrm{PVH})$ se considera una de las principales causas del cáncer cervical, aunque la mayor parte de las pruebas epidemiológicas proceden de estudios retrospectivos de casos y controles, que no aportan información sobre la dinámica de la exposición acumulada o persistente al virus. En este estudio se analizó el riesgo de neoplasia cervical relacionado con la persistencia de la infección por PVH.

Para ello se utilizaron los datos de un estudio longitudinal (Ludwig-McGill) sobre la evolución natural de la infección por PVH y de la neoplasia cervical en mujeres de bajo nivel socioeconómico residentes en la ciudad de São Paulo, Brasil. Entre noviembre de 1993 y marzo de 1997 se seleccionaron 2528 mujeres que cumplían los criterios de inclusión: tener edades comprendidas entre los 18 y los 60 años; residir de forma permanente en la ciudad de São Paulo; no estar embarazadas ni tener proyectado un embarazo en los 12 meses siguientes; tener útero intacto y no tener prevista la realización de una histerectomía; no haber utilizado medicaciones vaginales en los 2 días anteriores, y no haber recibido tratamientos cervicales con electrocoagulación, crioterapia o conización en los 6 meses anteriores.

Todas las participantes fueron examinadas a intervalos cuatrimestrales durante el primer año y a intervalos semestrales de ahí en adelante. En cada una de estas consultas se obtuvieron muestras cervicales para citología de Papanicolaou y detección de PVH. En los informes citopatólogicos se utilizó el sistema de clasificación de Bethesda. La presencia de $\mathrm{ADN}$ de los $\mathrm{PVH}$ se detectó mediante la reacción en cadena de la polimerasa. En una entrevista directa, se obtuvo información sobre los factores de riesgo de infección por $\mathrm{PVH}$ y neoplasia cervical (datos sociodemográficos, salud reproductiva, prácticas sexuales, consumo de tabaco y dieta). El seguimiento se prolongó hasta junio de 2000, la

\footnotetext{
1 El diseño y la metodología del estudio han sido descritos detalladamente en Franco E, Villa L, Rohan T, Ferenczy A, Petzl-Erler M, Matlashewski G. Design and methods of the Ludwig-McGill longitudinal study of the natural history of human papillomavirus infection and cervical neoplasia in Brazil. Rev Panam Salud Publica/Pan Am J Public Health 1999;6:223-233.
}

aparición de lesiones escamosas intraepiteliales, la muerte o la pérdida de seguimiento, según lo que ocurriera antes. ${ }^{1}$

En este análisis se incluyeron las 1611 mujeres sin lesiones citológicas en el primer examen y que disponían de resultados de las pruebas de detección de $\mathrm{PVH}$ en las dos primeras consultas. La proporción de casos que acudieron a las visitas programadas fue del $89 \%$ a los 12 meses, del $84 \%$ a los 24 , del $79 \%$ a los 36 , del $74 \%$ a los 48 , y del $69 \%$ a los 60 .

La tasa de incidencia de lesiones escamosas intraepiteliales fue de 0,73 por 1000 meses-mujer (intervalo de confianza del 95\% [IC95]: 0,5 a 0,9) en las mujeres sin infección por PVH en las dos primeras consultas, frente a 8,68 (IC95: 2,3 a 15,1) en las mujeres con infección por PVH de tipo 16 ó 18 persistente en ambas consultas. En comparación con las mujeres negativas para tipos oncógenos de PVH en las dos consultas iniciales, el riesgo relativo (RR) de lesiones intraepiteliales escamosas fue de 10,19 (IC95: 5,9 a 17,6) en aquellas con infecciones persistentes por algún tipo oncógeno de PVH. A su vez, el RR equivalente de lesiones intraepiteliales escamosas de alto grado fue de 11,67 (IC95: 4,1 a 33,3). Los RR fueron considerablemente mayores en las mujeres con infecciones persistentes por PVH de los tipos 16 ó 18.

Estos resultados corroboran los de otros estudios que han implicado a las infecciones persistentes por $\mathrm{PVH}$, en particular por tipos oncógenos $\mathrm{y}$, sobre todo, por los tipos 16 y 18, como causa de las lesiones intraepiteliales escamosas. (Schlecht NF, Kulaga S, Robitaille J, Ferreira S, Santos M, Miyamura RA, et al. Persistent human papillomavirus infection as a predictor of cervical intraepithelial neoplasia. JAMA 2001;286:3106-3114.)

\section{Relación entre las características del consumo de alcohol y la tensión arterial}

Las enfermedades cardiovasculares constituyen la principal causa de muerte en los países industrializados. Varios estudios han revelado que los acontecimientos cardiovasculares son más frecuentes los lunes que el resto de los días de la semana, fenómeno para el cual se han propuesto varias explicaciones, entre ellas el estrés que supone la 
vuelta al trabajo. Sin embargo, apenas se han estudiado las variaciones semanales de la magnitud de los factores de riesgo cardiovascular, como la tensión arterial (TA), que también podrían contribuir.

El consumo de alcohol está relacionado con la TA. En Francia, dicho consumo es constante a lo largo de la semana, mientras que en Irlanda del Norte tiene lugar, en su mayor parte, durante el viernes y el sábado. Además, se ha demostrado que los efectos de la ingesta aguda de alcohol son diferentes de los de la ingesta regular. Por consiguiente, los autores de este estudio se propusieron determinar si las características de la ingesta de alcohol en Irlanda del Norte y Francia tienen una influencia diferente sobre la TA.

Para ello utilizaron los datos del Estudio Epidemiológico Prospectivo del Infarto de Miocardio (Prospective Epidemiological Study of Myocardial Infarction: PRIME), en el que participaron tres centros de Francia (Lille, Estrasburgo y Toulouse) y uno de Irlanda del Norte (Belfast). Se estudió la relación entre el consumo de alcohol y la TA de lunes a viernes en varones sanos de edad mediana (50 a 59 años).

Se obtuvieron datos demográficos, socioeconómicos y sobre la dieta, el consumo de tabaco, alcohol y drogas, los antecedentes personales y familiares, y la actividad física. En el cuestionario sobre el consumo de alcohol se registró el consumo medio de vino, cerveza, cidra y licores cada día de la semana, expresado en $\mathrm{mL} /$ día de etanol puro. La TA se midió, en sedestación y tras 5 minutos de reposo, con un aparato automático (Spengler SP9). Las personas con antecedentes de angina de pecho, infarto del miocardio y posible cardiopatía coronaria fueron excluidas del análisis, al igual que las tratadas por hipertensión o dislipidemia. Las comparaciones univariadas entre los países se efectuaron con las pruebas de la $\chi^{2}$ o de la $t$ de Student, y las multivariadas con un modelo lineal general. Se consideraron significativos los valores de $P<0,05$.

De las 10595 personas inicialmente seleccionadas, 2418 fueron excluidas por los motivos antes mencionados. De las 8177 restantes (5 926 de Francia y 2251 de Irlanda del Norte), 6730 refirieron consumir alcohol al menos una vez a la semana (5 363 en Francia y 1367 en Irlanda del Norte).

Hubo varias diferencias significativas entre los bebedores franceses e norirlandeses: los primeros estaban casados con más frecuencia y tenían más edad, mayor índice de masa corporal, mayor nivel educacional, menor frecuencia cardíaca, menor consumo de tabaco, menor consumo de alcohol total, cerveza y licores, y mayor consumo de vino.

El consumo medio de alcohol fue ligeramente mayor en los norirlandeses que en los franceses ( $325 \pm 333$, frente a $317 \pm 249 \mathrm{~mL} /$ semana), aunque la diferencia fue estadísticamente significativa $(P<$ 0,01; prueba de Kruskal-Wallis). En los franceses, el consumo total de alcohol estaba distribuido uniformemente a lo largo de la semana, mientras que en los norirlandeses el $66 \%$ de dicho consumo tenía lugar los viernes y sábados.

Después de ajustar los datos por edad, índice de masa corporal, frecuencia cardíaca, número de años de escolaridad, estado civil, actividad laboral y consumo de tabaco, los norirlandeses tenían una TA sistólica (TAS) significativamente mayor y una TA diastólica (TAD) significativamente menor que los franceses. Además, tanto la TAS como la TAD presentaron diferencias significativas según el día e interacciones significativas entre el país y el día, incluso después de ajustar los resultados en función del consumo total de alcohol. En los norirlandeses bebedores, la TAS y la TAD fueron más elevadas el lunes y disminuyeron desde entonces hasta el viernes, mientras que en los franceses las cifras de TA fueron constantes a lo largo de toda la semana; en los no bebedores no se observaron relaciones similares. En los norirlandeses, la TAS y la TAD estaban correlacionadas de forma significativa con la cantidad media de alcohol consumida en los 3 y 4 días anteriores, mientras que en los franceses la relación entre la cantidad de alcohol consumida y la TAS y la TAD no mostró variaciones en función del intervalo de tiempo.

Estos resultados llevan a los autores a concluir que el gran consumo de alcohol concentrado en los fines de semana tiene un efecto adverso sobre la TA que no se observa cuando el consumo de alcohol está distribuido uniformemente a lo largo de toda la semana. Estos resultados concuerdan con los de otros estudios que han revelado que el diagnóstico de hipertensión arterial es más frecuente los lunes en los bebedores del fin de semana que en los bebedores con un consumo más uniforme a lo largo de toda la semana. Otros factores que no se investigaron en este estudio, pero que podrían estar relacionados con el consumo de alcohol durante el fin de semana, son el consumo de tabaco y de comidas saladas, o los efectos del consumo de cerveza no relacionados directamente con el alcohol, como el hiperaldosteronismo secundario a la hiponatremia inducida por la hipoosmolalidad de la cerveza. Por otra parte, los resultados obtenidos apoyan la hipótesis de que, debido al aumento de la TA, la gran ingesta de alcohol durante los fines de semana podría explicar, al menos de forma parcial, la mayor incidencia de infartos de miocardio que se observa los lunes. (Marques-Vidal P, Arveiler D, Evans A, Amouyel P, Ferrières J, Ducimetière P. Different alcohol drinking and blood pressure relationships in France and Northern Ireland: The PRIME Study. Hypertension 2001;38:1361-1366.) 
Los suplementos de vitamina A reducen los efectos adversos de la infección por VIH-1, de la malaria y de las diarreas infecciosas sobre el crecimiento infantil

El retraso del crecimiento y el déficit de vitamina A siguen siendo importantes problemas de salud pública en los lactantes y preescolares de los países en desarrollo. Algunos estudios observacionales han indicado que la vitamina A tiene un papel fundamental en el crecimiento, pero los ensayos clínicos han proporcionado resultados contradictorios que no permiten demostrar claramente una relación causal. Datos recientes indican que la aparente ausencia de efectos de la vitamina A sobre el crecimiento podría deberse a otros factores que afectan tanto al crecimiento como a la respuesta a los suplementos de esta vitamina, entre ellos las infecciones. Sin embargo, sigue existiendo un gran desconocimiento acerca de los efectos de los suplementos de vitamina A sobre el crecimiento de los niños con enfermedades infecciosas. La infección por el virus de la inmunodeficiencia humana (VIH), la malaria y las enfermedades diarreicas se asocian con un aumento de la prevalencia del déficit de vitamina A. Los autores de este estudio se propusieron investigar si los suplementos de vitamina A mejoran los efectos adversos de estas infecciones sobre el crecimiento infantil.

Para ello, entre abril de 1993 y marzo de 1977 se realizó un estudio aleatorizado, doblemente enmascarado, controlado con placebo, en 687 niños de 6 a 60 meses de edad ingresados con neumonía en un hospital de Dar es Salaam, Tanzanía. El estudio se diseñó para investigar los efectos de los suplementos de vitamina A sobre la gravedad de la neumonía durante la hospitalización y sobre la morbilidad, mortalidad y crecimiento posteriores al alta. Se excluyeron los niños con signos y síntomas oculares de déficit de vitamina $\mathrm{A}$, peso según la edad $<60 \%$ de la mediana de referencia y presencia de sarampión, tuberculosis pulmonar, difteria o tos ferina, así como los que habían recibido suplementos de vitamina $\mathrm{A}$ en $\operatorname{los} 4$ meses anteriores.

Aleatoriamente, los niños recibieron 200000 UI de vitamina A (60 mg de retinol en forma de retinilpalmitato) o placebo. El tratamiento asignado se administró en cuatro ocasiones: los dos primeros días del ingreso y 4 y 8 meses después del alta. Los menores de 12 meses recibieron la mitad de la dosis. Prácticamente el $100 \%$ de los niños tomaron las dos primeras dosis, administradas en el hospital, el $75 \%$ la tercera y el $74 \%$ la cuarta.

La talla, el peso y el perímetro del brazo se midieron mensualmente durante los 12 meses siguientes a la hospitalización inicial. La morbilidad se vigiló cada 15 días, prestando especial atención a las infecciones respiratorias y gastrointestinales. Se obtuvieron muestras de sangre de todos los casos en la consulta inicial, y de parte de ellos a los 4 y a los 8 meses. Las muestras se utilizaron para determinar parámetros hematológicos y detectar la presencia de malaria o infección por el VIH.

El análisis se efectuó por intención de tratar, con la participación de todos los niños en los que se realizaron al menos dos mediciones antropométricas más de 14 días después del alta del hospital. Las variables de eficacia primarias fueron el aumento del peso y de la talla en los 12 meses posteriores al ingreso.

En 554 de los 687 participantes se efectuaron las dos mediciones antropométricas necesarias para que pudieran ser incluidos en el análisis. La duración mediana del período de seguimiento y el número mediano de mediciones por niño fueron idénticas en ambos grupos: 351 días y 10 mediciones/ niño, respectivamente. Tampoco hubo diferencias significativas entre los grupos con respecto a la distribución de las características basales. El 24\% de los niños (115) fueron diagnosticados de malaria, por Plasmodium falciparum en prácticamente todos, y el 9\% (47), de infección por VIH. La prevalencia del retraso del crecimiento y de la emaciación en el examen inicial fue del 30,7\% y $8,6 \%$, respectivamente.

Los suplementos de vitamina A no tuvieron un efecto global significativo sobre el aumento de la talla ni del peso al cabo de 1 año, ni antes ni después de ajustar los datos en función de la presencia de malaria, infección por VIH o anemia grave, del bajo perímetro del brazo, de la calidad del suministro de agua, del número de enseres del domicilio ni de la estación en la que se efectuó la aleatorización. En los 372 niños que no presentaban retraso del crecimiento en el examen basal, los suplementos de vitamina A se asociaron con una reducción casi significativa (23\%; intervalo de confianza del $95 \%$ [IC95]: -4 a $44 \% ; P=0,09$ ) del riesgo de retraso del crecimiento en el año siguiente. Este resultado se mantuvo después de controlar el efecto de la edad, de la malaria y la infección por VIH, del perímetro del brazo y del número de enseres del domicilio. En los 491 niños sin emaciación en el examen inicial, los efectos de la vitamina A sobre el riesgo de emaciación en el año siguiente no fueron significativos antes ni después del ajuste (riesgo relativo [RR] no ajustado: 0,88; IC95: 0,52 a 1,50).

En los niños de menos de 18 meses infectados por el VIH, los suplementos de vitamina A produjeron un significativo aumento de la talla. Cuatro meses después de la primera dosis, estos niños habían crecido 2,8 cm (IC95: 1,0 a 4,6) más que los que recibieron placebo; en los no infectados por el VIH no se observó este efecto (diferencia a los 4 meses: $-0,2 \mathrm{~cm}$; IC95: $-0,8$ a 0,5). Los menores de 12 meses 
con malaria en el examen inicial y tratados con vitamina A tuvieron un aumento de peso anual de $747 \mathrm{~g}$ (IC95: 71 a 1423) más que los tratados con placebo; en los niños sin malaria, el efecto no fue significativo (-57 g; IC95: -461 a 348). Estos resultados no cambiaron tras los ajustes en función de varios indicadores socioeconómicos y del estado nutricional al inicio del estudio. En los niños cuyas casas tenían malos suministros de agua, la vitamina $\mathrm{A}$ también mejoró el crecimiento en estatura $(0,8 \mathrm{~cm} / \mathrm{año}$; IC95: 0 a 1,5) y, además, se asoció con una reducción del 36\% (IC95: 3 a 58\%) en el riesgo de retraso del crecimiento; en aquellos cuyas casas disponían de agua corriente no se observó ninguno de estos dos efectos. El aumento de peso fue mayor en los niños con perímetro braquial inferior al percentil 25 (458 g/año; IC95: 1 a 905), pero la vitamina A no proporcionó ningún beneficio en aquellos con mayor perímetro. Los suplementos de vitamina A eliminaron prácticamente el riesgo de retraso del crecimiento asociado con los episodios de diarrea persistente (duración > 14 días) (RR: 1,0; IC95: 0,3 a $1,3)$, pero no modificaron el riesgo de retraso del crecimiento ni de emaciación asociado con las infecciones respiratorias.

De acuerdo con los resultados obtenidos, los autores concluyen que la administración cuatrimestral de suplementos de vitamina $\mathrm{A}$ a los niños de menos de 5 años es una medida de bajo costo que puede ser eficaz para mejorar su crecimiento en poblaciones con una elevada incidencia de enfermedades infecciosas como la infección por VIH, la malaria, la diarrea persistente y la disentería. En este estudio, los suplementos mejoraron el crecimiento en estatura de los lactantes infectados por el VIH y el crecimiento ponderal de los lactantes con malaria, además de reducir el riesgo de retraso del crecimiento asociado con la diarrea persistente. (Villamor E, Mbise R, Spiegelman D, Hertzmark E, Fataki M, Peterson KE, et al. Vitamin A supplements ameliorate the adverse effect of HIV-1, malaria, and diarrheal infections on child growth. Pediatrics 2002;109(1):e6 [Publicación previa en versión electrónica. Disponible en: http://www.pediatrics.org/cgi/content/full/ 109/1/e6 Acceso el 22 de enero de 2002].)

\section{Cumplimiento del tratamiento antirretrovírico en pacientes con infección por $\mathrm{VIH} /$ sida}

El cumplimiento del tratamiento es muy importante, principalmente cuando se trata de enfermedades crónicas. En el caso de la infección por el virus de la inmunodeficiencia humana (VIH) o del sida, el uso incorrecto de los antirretrovíricos se asocia no solo con el fracaso del tratamiento, sino tam- bién con la aparición de cepas resistentes a los fármacos. Los estudios realizados hasta la fecha revelan que el cumplimiento de los tratamientos antirretrovíricos oscila entre el 40 y el $80 \%$ y que para mantener la carga vírica indetectable y obtener aumentos significativos del recuento de linfocitos $\mathrm{CD}^{+}$, es necesario que sea superior al $90 \%$. Por otra parte, la evaluación del cumplimiento del tratamiento antirretrovírico resulta difícil, porque ninguno de los métodos existentes (autoinforme, supervisión electrónica de los frascos de medicamentos y determinación de la concentración sérica de los fármacos) es totalmente satisfactorio. Debido a la existencia de escasos datos sobre el cumplimiento de los tratamientos antirretrovíricos en Brasil, se realizó el presente estudio, cuyo objetivo consistió en cuantificar dicho cumplimiento e identificar los principales factores predictivos y las causas de incumplimiento.

El estudio, de tipo transversal, se realizó entre abril y junio de 1999 en una muestra de pacientes con infección por VIH o sida en un centro de la Universidad Federal de Minas Gerais especializado en enfermedades infecciosas. Los criterios de inclusión fueron: tener edad superior a 18 años, estar diagnosticado de sida según los criterios utilizados por el Ministerio de Salud y estar en tratamiento en el centro. De los 150 pacientes seleccionados, 30 se negaron a participar o no contestaron a la petición de participación.

Los datos para evaluar el cumplimiento del tratamiento se obtuvieron a partir de cuestionarios a los pacientes, de las fichas de dispensación de la farmacia del centro, de diarios rellenados por los pacientes y de las historias clínicas. En los cuestionarios se obtuvo información sobre las variables sociodemográficas, modo de infección, utilización de los antirretrovíricos el día anterior y en los últimos 3, 15 y 30 días y los motivos que llevaron a los pacientes a no utilizar los medicamentos; se consideró que había adherencia al tratamiento cuando su cumplimiento fue superior al 90\% en los últimos 3 días. En las fichas de dispensación de la farmacia se investigaron las dos o tres últimas retiradas de medicamentos y se consideró que había cumplimiento del tratamiento cuando el retraso de la retirada fue inferior a 6 días, para períodos de 60 días, o a 9 días, para períodos de 90 días. Los diarios fueron rellenados por una submuestra aleatoria de 24 pacientes $(20 \%)$, cuyas características sociodemográficas eran similares a las del resto de la muestra; durante un mes, anotaron diariamente el horario y las dosis consumidas de cada uno de los antirretrovíricos prescritos; en este caso, el cumplimiento se definió como el uso de al menos un $90 \%$ de los medicamentos prescritos ese mes. De las historias clínicas se obtuvo el valor de la carga vírica, con el fin de identificar una posible asociación entre 
el incumplimiento del tratamiento y la persistencia de la carga vírica por encima de los límites de detección $(80$ copias $/ \mathrm{mL})$.

Los pacientes tenían una media de 35,5 años, $72,5 \%$ eran varones, $37,8 \%$ habían tenido relaciones heterosexuales sin preservativos y $31,1 \%$, relaciones homosexuales sin preservativos. La duración media del uso de antirretrovíricos fue de 12 meses ( 1 a 88) y la mediana de 8 meses. Predominaron los tratamientos con tres o más fármacos $(70,8 \%)$ sobre los tratamientos con dos fármacos $(29,2 \%)$. Las causas más frecuentes que, según los pacientes, los llevaron a no tomar el tratamiento fueron los efectos colaterales de las medicaciones $(20,5 \%)$ y las dificultades para seguir la posología recomendada, bien por la inoportunidad de las tomas $(19,9 \%)$ o por olvido $(17,3 \%)$. Entre los efectos colaterales, los más frecuentes fueron los gastrointestinales $(51,6 \%)$, los generales $(30,6 \%)$, los neurológicos $(8,8 \%)$ y los dermatológicos $(8,8 \%)$.

Los pacientes que cumplieron el tratamiento fueron $74 \%$ en los últimos 3 días según los cuestionarios, 76,5\% según las fichas de dispensación de la farmacia (solo hubo 85 fichas válidas para este análisis, lo cual representa un 70,8\%), y 70,8\% según los diarios. Los coeficientes de variación de las tres estimaciones fueron similares.

Se analizaron las siguientes variables que podrían predecir el cumplimiento del tratamiento antirretrovírico: edad, sexo, escolaridad, color de la piel, empleo, renta mensual, duración de la infección por VIH, modo de infección, duración del uso de antirretrovíricos y esquema terapéutico. En los dos análisis multivariados (uno con los datos de los cuestionarios y otro con los datos de las fichas de dispensación de la farmacia) se incluyeron las variables que en el análisis univariado presentaron un valor $P \leq 0,15$. En el primero se incluyeron la edad, el sexo, la escolaridad y la renta. En el modelo final, las variables que contribuyeron a explicar el incumplimiento del tratamiento fueron la edad inferior a 40 años y la escolaridad inferior a 4 años. En el segundo se incluyeron la duración del uso de antirretrovíricos, el sexo y la escolaridad. En el modelo final, las variables que contribuyeron a explicar el incumplimiento del tratamiento fueron la escolaridad inferior a 4 años y la duración del tratamiento antirretrovírico inferior a 8 meses. Por último, la relación entre el cumplimiento del tratamiento y la persistencia de la carga vírica por encima del límite de detección se analizó en 75 pacientes $(62,5 \%)$. El porcentaje de pacientes con carga vírica indetectable fue del $46 \%$ entre los que cumplían el tratamiento, y del $24 \%$ entre los que no lo cumplían; la diferencia no fue estadísticamente significativa.

De acuerdo con estos resultados, los autores concluyen que, para mejorar el cumplimiento del tratamiento antirretrovírico, es necesario utilizar esquemas terapéuticos mejor tolerados, con menos efectos colaterales, y ayudar a los pacientes a que no se olviden de tomar la medicación. (Júnior LL, Greco DB, Carneiro M. Avaliação da aderência aos anti-retrovirais em pacientes com infecção pelo HIV/Aids. Rev Saude Publica 2001;35:495-501.)

\section{Los anticonceptivos orales y el riesgo de infarto del miocardio}

El consumo de anticonceptivos orales (AO) constituye, según varios estudios, un factor de riesgo de trombosis tanto venosa como arterial. Con el fin de reducir esos riesgos, se han efectuado varias modificaciones, tales como la reducción de las dosis de estrógenos o la sustitución de unos progestágenos por otros. Los AO que contienen los llamados progestágenos de tercera generación (desogestrel o gestodeno) se han asociado con una duplicación del riesgo de trombosis venosa en comparación con otros $\mathrm{AO}$ combinados, pero, por otro lado, se ha señalado que podrían proteger frente al infarto del miocardio (IM). Los autores de este estudio investigaron la asociación entre el consumo de $\mathrm{AO}$ y el riesgo de IM en función del tipo de progestágenos (desogestrel o gestodeno, frente a levonorgestrel), de la dosis de estrógenos y de la presencia o ausencia de mutaciones protrombóticas (mutación G1691A del gen del factor V y mutación G20210A del gen de la protrombina).

En este análisis se utilizaron los datos del estudio sobre el riesgo de trombosis arterial en relación con el consumo de AO (Risk of Arterial Thrombosis in Relation to Oral Contraceptives Study: RATIO), un estudio de casos y controles basado en la población y de ámbito nacional, realizado en Holanda, en el que participaron 248 mujeres de 18 a 49 años que habían sufrido su primer IM entre 1990 y 1995, y 925 controles sin IM, seleccionadas aleatoriamente y apareadas con los casos según la edad, el año del acontecimiento índice y la zona de residencia. Todas la participantes proporcionaron información sobre sus características sociodemográficas, el consumo de $\mathrm{AO}$ y los principales factores de riesgo cardiovascular (antecedentes personales de hipertensión, diabetes, hipercolesterolemia y consumo de tabaco, y antecedentes familiares de enfermedades cardiovasculares). En 217 pacientes y 763 controles se procedió a la identificación de la presencia o ausencia de las mencionadas mutaciones de los genes del factor $\mathrm{V}$ y de la protrombina mediante la reacción en cadena de la polimerasa. La razón de los productos cruzados (odds ratio: OR) de la relación entre el consumo de $\mathrm{AO}$ y el IM y su intervalo de 
confianza del 95\% (IC95) se calculó mediante análisis de regresión logística incondicional. Los datos se ajustaron en función de la edad, del año del acontecimiento índice y de la zona de residencia, así como de los posibles factores de confusión (consumo de tabaco, hipercolesterolemia, diabetes, hipertensión, obesidad, antecedentes familiares de enfermedad cardiovascular, nivel educacional y consumo de alcohol). También se repitieron los análisis después de excluir a las mujeres con los principales factores de riesgo cardiovascular.

En comparación con las mujeres que no usaban $\mathrm{AO}$, el riesgo de IM fue dos veces mayor en aquellas que utilizaban algún tipo de $\mathrm{AO}$ combinado (OR ajustada: 2,0; IC95: 1,5 a 2,8). La OR ajustada fue de 2,5 (IC95: 1,5 a 4,1) en las que usaban $\mathrm{AO}$ de segunda generación y de 1,3 (IC95: 0,7 a 2,5) en las que usaban $\mathrm{AO}$ de tercera generación. En la comparación directa de los AO que contenían $30 \mu \mathrm{g}$ de etinilestradiol y levonorgestrel, desogestrel o gestodeno, la OR fue de 0,5 (IC95: 0,2 a 1,1) en las consumidoras de $\mathrm{AO}$ de tercera generación, en comparación con las consumidoras de $\mathrm{AO}$ de segunda generación. Las OR no mostraron diferencias significativas en función de las dosis de estrógenos. Entre las mujeres que consumían $\mathrm{AO}$, las OR fueron similares en las que presentaban mutaciones protrombóticas (OR: 1,9; IC95: 0,6 a 5,5) y en las que no las presentaban (OR: 2,1 IC95: 1,5 a 3,0).

En resumen, los resultados de este estudio revelan que el riesgo de IM está aumentado en las mujeres que toman $\mathrm{AO}$ de segunda generación; en las que toman $\mathrm{AO}$ de tercera generación, los resultados no fueron concluyentes, pero indicaron que el riesgo parece ser menor que en el grupo anterior. Asimismo, se comprobó que el riesgo de IM en las mujeres que toman $\mathrm{AO}$ es similar en las que tienen mutaciones protrombóticas y las que no las tienen. Aunque el riesgo de $\mathrm{IM}$ en las consumidoras de $\mathrm{AO}$ es pequeño en términos absolutos, tiene importantes repercusiones sobre la salud de la mujer, dado que un 35 a $45 \%$ de las mujeres en edad reproductiva toman $\mathrm{AO}$. Los autores concluyen que antes de prescribir $\mathrm{AO}$ a una mujer se deben investigar sus factores de riesgo convencionales de enfermedad cardiovascular y que el consejo más importante que se les debe dar es que dejen de fumar. (Tanis BC, van den Bosch MAAJ, Kemmeren JM, Cats VM, Helmerhorst FM, Algra A, et al. Oral contraceptives and the risk of myocardial infarction. N Engl J Med 2001;345:1787-1793.)

\section{El consumo de alcohol y el riesgo de demencia}

Varios estudios han indicado que el consumo de grandes cantidades de alcohol tiene un efecto neurotóxico. Por otro lado, el consumo ligero a moderado parece asociarse con una disminución del riesgo de cardiopatía coronaria, accidente cerebrovascular isquémico y mortalidad total, tanto en hombres como en mujeres de edad avanzada. Dado que cada vez hay más pruebas de que las enfermedades vasculares se asocian con el deterioro cognitivo y la demencia, dicho consumo también podría reducir el riesgo de demencia en general, y de enfermedad de Alzheimer en particular. En este estudio se investigó esta hipótesis y si el efecto depende del tipo de bebida alcohólica.

Se investigó la relación entre el consumo de alcohol y el riesgo de demencia en los participantes en el Estudio de Rotterdam, un estudio de cohorte prospectivo basado en la población, en el que participaron 7983 personas de 55 años o más residentes en un suburbio de Rotterdam, Holanda, incluidas las que vivían en instituciones. En este análisis se incluyeron todas las que no padecían demencia en el examen inical, realizado entre 1990 y 1993, y proporcionaron datos completos sobre su consumo de alcohol $(n=5$ 395). Mediante dos exámenes de seguimiento, realizados en 1993-94 y 1997-99, y un extenso sistema de monitorización, se obtuvieron datos de seguimiento de prácticamente todas estas personas $(99,7 \%)$ hasta el final de 1999. Los diagnósticos de demencia se establecieron de acuerdo con criterios bien reconocidos (NINCDS-ADRDA, NINDS-AIREN, DSM-III-R). La genotipificación de la apolipoproteína E (APOE) se realizó mediante la reacción en cadena de la polimerasa. Los homocigóticos y heterocigóticos para el alelo $A P O E^{*} 4$ se combinaron en una sola categoría. Para comparar el riesgo de padecer demencia entre las personas que consumían alcohol habitualmente y las que no lo hacían, se realizó un análisis de regresión de Cox, ajustado por edad, sexo, diabetes, tensión arterial sistólica, nivel educacional, consumo de tabaco e índice de masa corporal. Los análisis se repitieron considerando separadamente la enfermedad de Alzheimer y la demencia vascular. Mediante análisis estratificados, también se investigó la posible modificación del efecto por el sexo, la edad, el consumo de tabaco, el nivel educacional y el genotipo de la APOE. Por último, se analizó el riesgo de demencia y de los subtipos de demencia según el tipo de bebidas alcohólica (vino, cerveza, licores y vinos fortificados).

Durante los 32341 años-persona de seguimiento (seguimiento medio de 6 años), 197 participantes sufrieron demencia, lo cual representa una tasa de incidencia de 6,1/1000 años-persona. Se diagnosticó enfermedad de Alzheimer en 146 (74\%; 12 con y 134 sin enfermedad cerebrovascular), demencia vascular en $29(15 \%)$ y otros tipos de demencia en $22(11 \%)$. 
El consumo mediano de alcohol fue de 0,29 vasos/día (recorrido intercuartílico [RIC]: 0,01 a $1,24)$, siendo mayor en los hombres (0,87; RIC: 0,15 a 2,24$)$ que en las mujeres $(0,11$; RIC: 0,00 a 0,73$)$. Los hombres consumieron principalmente cerveza y licores, y las mujeres, vinos fortificados.

El consumo de alcohol ligero a moderado (1 a 3 copas al día) se asoció con un riesgo significativamente menor de demencia en general (razón de riesgo instantáneo [hazard ratio: $\mathrm{HR}$ ]: 0,58; intervalo de confianza del 95\% [IC95\%]: 0,38 a 0,90]) y de demencia vascular (HR: 0,29; IC95\%: 0,09 a 0,93). El efecto fue más pronunciado en los hombres (HR ajustada por edad: 0,39; IC95\%: 0,21 a 0,72) que en las mujeres (HR ajustada por edad: 0,80; IC95\%: 0,45 a 1,45). Los análisis en función del subtipo de demencia revelaron que el efecto del alcohol estaba presente sobre todo en la demencia vascular. No se obtuvo ninguna prueba de que el riesgo de demencia o de los diferentes subtipos de demencia variara en función del tipo de bebidas alcohólicas consumidas. El consumo de alcohol ligero a moderado se asoció con un menor riesgo de demencia vascular tanto en presencia como en ausencia del alelo $A P O E^{*} 4$, aunque los intervalos de confianza eran amplios debido al pequeño tamaño de la muestra. En el caso de las demencias en general y de la demencia de Alzheimer en particular, la reducción del riesgo asociada al consumo de alcohol se consiguió con menores cantidades de alcohol en los portadores del alelo $A P O E^{*} 4$.

En resumen, el presente estudio indica que el consumo ligero a moderado de alcohol se asocia con una disminución del riesgo de demencia en personas de 55 años o más y que el efecto no parece depender del tipo de bebidas alcohólicas consumidas. (Ruitenberg A, van Swieten JC, Witteman JCM, Mehta KM, van Duijn CM, Hofman A, et al. Alcohol consumption and risk of dementia: the Rotterdam Study. Lancet 2002;359:281-286.)

\begin{tabular}{|c|}
\hline $\begin{array}{l}\text { Segunda Conferencia Internacional sobre Equidad } \\
\text { Second International Conference on Equity: Research in the Service of Policy } \\
\text { and Advocacy for Health and Health Services }\end{array}$ \\
\hline $\begin{array}{l}\qquad \begin{array}{l}\text { Fechas: } 14 \text { a } 16 \text { de junio de } 2002 \\
\text { Lugar: }\end{array} \\
\text { Toronto, Canadá } \\
\text { La International Society for Equity in Health [ISEqQH, Sociedad Internacional para la Equidad en } \\
\text { ver la equidad mediante actividades de investigación, educación y divulgación. Entre sus fines específicos } \\
\text { figuran: 1) facilitar el intercambio de información teórica y metodológica entre científicos en torno a la } \\
\text { equidad en materia de salud y en el ámbito de los servicios asistenciales; 2) fomentar las investigaciones } \\
\text { en este campo; 3) proveer una tribuna donde puedan expresar sus apreciaciones y conocimientos quie- } \\
\text { nes se interesan en la falta de equidad que se observa en los sistemas y servicios de salud, y 4) reforzar } \\
\text { Ios lazos entre organizaciones internacionales y regionales dedicadas a conseguir una mayor equidad en } \\
\text { estos ámbitos. } \\
\text { La International Society for Equity in Health es una organización no gubernamental sin fines de } \\
\text { Iucro financiada por aportes de sus miembros y por subvenciones de la OPS, la Fundación Rockefeller, la } \\
\text { Fundación Soros y la March of Dimes. } \\
\text { Información: } \\
\text { Correo electrónico: iseqh.exec@utoronto.ca } \\
\text { Teléfono: } 4169783763 \\
\text { Fax: } 4169783912\end{array}$ \\
\hline
\end{tabular}

